\title{
Campaign Scheduling and Analysis for the Gemini Planet Imager
}

\author{
Dmitry Savransky ${ }^{1}$, Bruce A. Macintosh ${ }^{1}$, James Graham ${ }^{2}$, Quinn M. \\ Konopacky ${ }^{3}$ and the GPI science team \\ ${ }^{1}$ Lawrence Livermore National Lab, Livermore, CA USA, \\ email: savransky1@llnl.gov \\ ${ }^{2}$ University of California Berkeley, Berkeley, CA USA \\ ${ }^{3}$ Dunlap Institute, University of Toronto, Toronto, Canada
}

\begin{abstract}
The Gemini Planet Image (GPI) is a new, high-contrast, exoplanet-imaging, facility instrument for the Gemini South observatory, scheduled to begin science observations in 2014 . The GPI Exoplanet Survey (GPIES) has been awarded 890 hours to image and spectrally and polarimetrically characterize young, giant planets within 100 parsecs of the solar system. In preparation for the survey, we have developed a framework for simulating GPI observations and generating end-to-end survey simulations. We present new extensions to this modeling effort and our latest results. We discuss systematic methods for scheduling the survey to ensure that the population of discovered planets is useful in constraining formation models and possibly distinguishing between gravitational collapse and core accretion as the primary formation mechanism.
\end{abstract}

Keywords. GPI, Exoplanets, Surveys, Survey modeling, Survey analysis

\section{GPI and GPIES Overview}

GPI is an advanced, precision AO system combined with a coronagraph and integralfield, near-infrared spectrograph designed to image self-luminous, young, giant planets (Macintosh et al. 2012). GPI currently achieves raw $(5 \sigma)$ contrasts of $2 \times 10^{-6}$ in 60 seconds in a single slice of an extracted $\mathrm{H}$ band data cube. Simulated, hour-long observing sequences, based on lab data with atmospheric phase plates, predict contrasts of $3 \times 10^{-7}$ with post-processing (Marois et al. 2010).

GPIES aims to produce the first robust census of giant planet populations in the $5-50$ AU range. We will observe 600 stars (see Fig. 1(a)) spanning spectral types A-M selected from published young association catalogs and a proprietary list - the result of thousands of hours of telescope observations in preparation for GPI - that adds several hundred new young $(<100 \mathrm{Myr},<75 \mathrm{pc})$ and adolescent $(<300 \mathrm{Myr},<35 \mathrm{pc})$ stars, which will allow our survey to probe in to the $5 \mathrm{AU}$ ice line. The range of separations studied by GPI is inaccessible to Doppler and transit techniques and will provide a new window into planet formation.

\section{Survey Simulation}

Surveys are automatically scheduled by maximizing the cost function:

$$
J=k_{1} p_{\text {det }}-k_{2} \frac{t_{\text {tot }}}{t_{\text {rem }}}
$$

where $p_{\text {det }}$ is the probability of detection (McBride et al. 2011), $t_{\text {tot }}$ is the time the target will be observable for the remainder of the survey, $t_{\text {rem }}$ is the time remaining, 

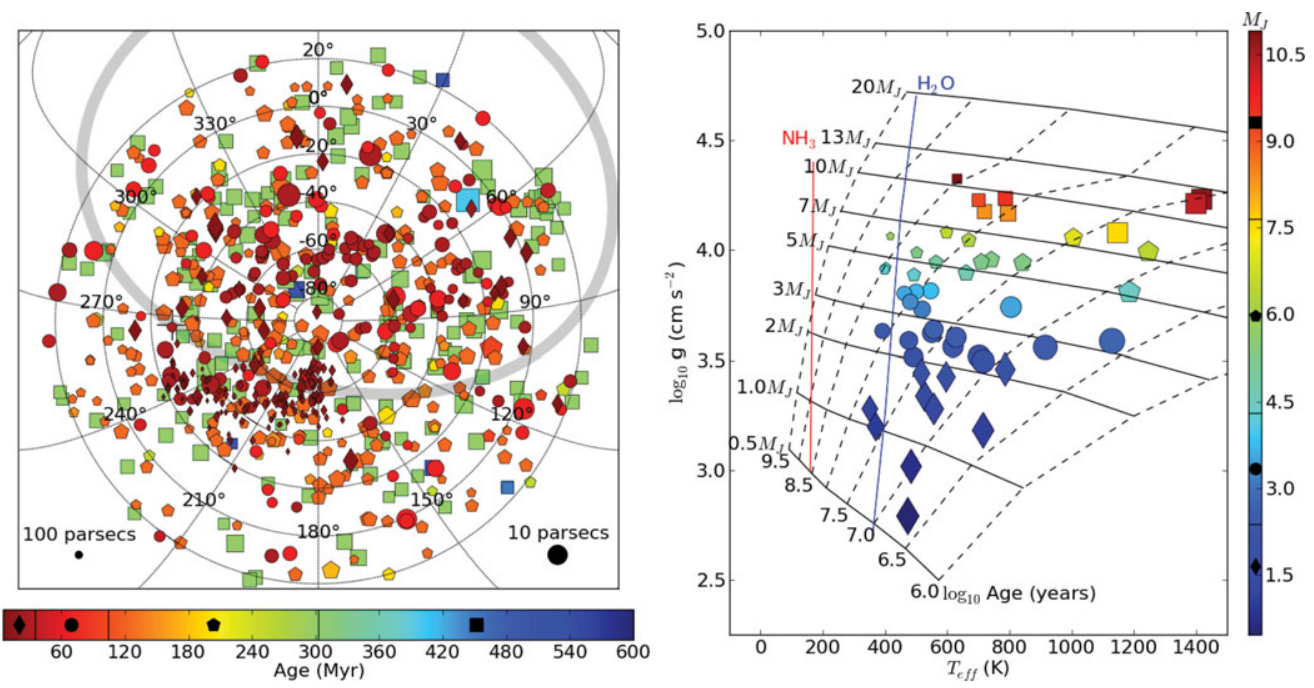

Figure 1. (a) GPIES targets, shaded by age and sized (inversely) by distance. Symbols represent ages in the bins marked on the colorbar. The grey band represents the galactic equator with the galactic center marked by the ' + '. (b) Gravity vs. effective temperature with evolutionary tracks (solid black lines) and isochrones (dashed lines) from Burrows et al. (2003). Points are simulated planets with size proportional to their radii and shaded by mass. Symbols represent masses in the bins marked on the colorbar.

and $k_{1}$ and $k_{2}$ are normalized weights. Followups are selected to maximize diversity in mass and semi-major axis space, using contrast as a proxy for mass and the projected separation as an estimate for the semi-major axis (Savransky et al. 2011). We produce thousands of sample surveys and analyze the ensembles to predict and optimize survey performance.

\section{Simulation Results}

Simulations show a yield of $24.8 \pm 4.8$ unique planet detections, on average, when all planets are modeled as cold start, and $39.6 \pm 6$ when all are modeled as hot start. We also find that the planets detected in the simulations span a sufficient space in surface gravity and effective temperature to make the sample useful in model selection (Fig. 1(b)) and that we are able to account for survey selection biases in fitting the distributions of masses and semi-major axes with average sample sizes. We also find that simulated astrometric data points for multiple observations of a subset of planets allow us to discriminate between different input distributions of eccentricities, so that the survey will be able to tell whether discovered orbits are predominantly circular or eccentric.

This work performed under the auspices of the U.S. Department of Energy by Lawrence Livermore National Laboratory under Contract DE-AC52-07NA27344.

\section{References}

Burrows, A., Sudarsky, D., \& Lunine, J. I. 2003, ApJ, 596, 587

Macintosh, B. A., et al. 2012, in: Proc. SPIE, Vol. 8446, 84461U

Marois, C., Macintosh, B., \& Véran, J. P. 2010, in: Proc. SPIE, Vol. 7736, 77361J

McBride, J., Graham, J., Macintosh, B., Beckwith, S., Marois, C., Poyneer, L., \& Wiktorowicz, S. 2011, PASP, 123, 692

Savransky, D., Cady, E., \& Kasdin, N. J. 2011, ApJ, 728, 66 